\title{
Das Schulprojekt Geschichtomat: Historische Orte mit digitalen Mitteln entdecken
}

• geschichtomat@igdj-hh.de, Institut für die Geschichte der deutschen Juden, Hamburg

eingereicht am: 03.06.2019, akzeptiert am: 26.08.2019

Wie kann jüdische Geschichte für Jugendliche „zeitgemäß“ und alltagsnah vermittelt werden? Am Beispiel des Hamburger Schulprojekts Geschichtomat soll versucht werden, darauf eine Antwort zu finden. Das Projekt verbindet Lokalgeschichte mit digitalen Medien, um die all-tägliche Umgebung der Jugendlichen in den Geschichtsunterricht einzubeziehen. Es regt Schüler/innen dazu an, Geschichte eigenständig zu erforschen und eigene Fragestellungen zu entwickeln. Ihre Eindrücke halten die Jugendlichen in Filmen fest, die sie in einem digitalen Stadtplan hochladen.

Keywords: Medienerziehung, Digitale Bildung, Lokalgeschichte, Jüdische Geschichte

\section{The school-project „Geschichtomat": Exploring historic sites with digital media tools}

How can history be taught in a modern and everyday life like way? Taking the student-driven project from Hamburg Geschichtomat as an example, this article tries to find an answer to this question. The project combines local history and media education to create a learning envi-ronment for the students within their local surrounding. It encourages students to discover history and to develop questions on their own. Their results are uploaded to the geschichtomat.de-website.

Keywords: media education, local history, Jewish history

\section{$1 \quad$ Einleitung}

„Think historically, act locally!“, unter diesem Motto werden Ressourcen in Museen, Bildungseinrichtungen und von Lehrkräften verwendet, in der Hoffnung Schüler/innen zum eigenen Forschen und sich Einbringen zu motivieren (Hunner 2011: 33). Die Lokalgeschichte scheint dabei ein vielversprechendes Mittel zu sein. Mit Methoden wie Oral History können Jugendliche in Verbindung zu ihren Mitmenschen treten. Sie sprechen mit Zeitzeuginnen und Zeitzeugen, können ihre eigenen Fragen entwickeln und stellen dadurch einen persönlichen Bezug zu diversen Themen her. Diese werden in der persönlichen Erzählung oftmals leichter verständlich. Dadurch kann sich im günstigsten Fall sogar ein Zugang zur „größeren“ Geschichte dahinter entwickeln (Hunner 2011: 40).

Doch auch zu einem zu erforschenden historischen Ort können Jugendliche einen Bezug besitzen. Vielleicht befindet sich dieser in der Nachbarschaft oder auf ihrem Nachhauseweg. Das Aufsuchen ist dann oftmals problemlos möglich. Stadtarchive, Gedenk- stätten und andere Institutionen besitzen gegebenfalls noch Originalquellen, mit denen gearbeitet werden kann und die in die Hand genommen werden können. Dies bietet viele Vorteile gegenüber einem Geschichtsbuch (John 2018: 77). Geschichte ist plötzlich nicht mehr abstrakt, sondern auf lokaler Ebene greifbar und bietet persönliche Berührungspunkte. Das Interesse der Schüler/innen an Geschichte könnte also bereits durch eine lokale Verknüpfung mit ihrem Alltag geweckt sein.

Doch so einfach wie dieser Zugang klingt, ist er vielfach nicht. Fest steht: Unterricht gelingt besser, wenn die Lebenswelt und die Erfahrungen der Schüler/innen mit einbezogen werden. Es darf aber nicht vergessen werden, dass Jugendliche sehr unterschiedlich mobil sind und ganz verschiedene Nahbereiche besitzen. Zudem gelingt es Schüler/innen problemlos Bauwerke, Denkmäler und andere historische Stätten auf ihrem Schulweg zu ignorieren und nicht wahrzunehmen. Deswegen muss historisches Geschehen immer erst vergegenwärtigt werden, um sich auch damit auseinandersetzen zu können. Ohne Anschub der 
Aufmerksamkeit und anleitende Hilfe begegnen Jugendliche Denkmälern und historischen Orten daher eher unbedarft. Vielfach werden sie pauschal als „alt“ eingeordnet. Der historische Kontext ist schlichtweg oftmals nicht bekannt (John 2018: $46 \mathrm{f}$.).

Lokalgeschichte zeichnet sich durch ihre Verbindung zum Lebensalltag aus und ist dadurch für Schüler/innen oftmals leichter nachvollziehbar (John 2018: 79). Eine Einbindung in den Geschichtsunterricht mit passender Anleitung und Hilfestellung ist deshalb sinnvoll. Reicht dies, um Geschichtsunterricht „zeitgemäß” zu gestalten und möglichst alle Jugendlichen anzusprechen?

Ein anderer Aspekt des Alltags der Jugendlichen darf nicht unterschätzt werden: die digitalen Medien. Immer wieder wird deswegen insbesondere von politischer Seite die Förderung eines reflektiertkritischen Umgangs mit digitalen Medien gefordert. Geschichte als Unterrichtsfach scheint dafür sehr gut geeignet. Die historisch-kritische Methode kann gut zur „historischen Medienkompetenz" beitragen. Eine Integration digitaler Medien in den Unterricht als Lerngegenstand, z. B. durch die Erstellung eigener digitaler Geschichtsprodukte, durch die Arbeit mit bereits vorhandenen, durch das Einüben von Recherchetechniken sowie die kritische Hinterfragung von Medienangeboten scheint deshalb sinnvoll (Schwabe 2018: 421).

Wenn Lokalgeschichte einen leichteren Zugang zu Geschichte bietet und digitale Medien gleichzeitig dem Lebensalltag der Jugendlichen entsprechen, lassen sich diese beiden Komplexe miteinander verbinden, um einen modernen, praxisnahen und interessanten Geschichtsunterricht für Schüler/innen zu gestalten?

Im Folgenden soll das Hamburger Schülerprojekt "Geschichtomat" vorgestellt und untersucht werden. Es unternimmt genau diesen Versuch: Lokalgeschichte mit digitalen Medien zu verknüpfen. Im Fokus des Projektes steht dabei die Vermittlung der jüdischen Geschichte in Hamburg, einem Bereich, der im Schulalltag so gut wie keine Rolle spielt (vgl. Behörde für Schule und Berufsbildung Freie und Hansestadt Hamburg 2014; Landesinstitut für Lehrerbildung Hamburg 2011).

Bei der Vermittlung der jüdischen Geschichte treten noch einmal spezielle Probleme auf. Im bundesdeutschen Schulalltag ist wenig Zeit sich mit jüdischer Geschichte und Kultur jenseits des Zivilisationsbruchs des Holocausts zu beschäftigen. Eine empirische Studie deutscher Schulbücher zeigt, dass jüdische Geschichte vorrangig in Verbindung mit dem Nationalsozialismus und dem Holocaust unterrichtet wird (Liepach et al. 2014: 15). Auch in anderen Epochen wird jüdische Geschichte meist mit
Verfolgungsgeschichte gleichgesetzt. Dies ist problematisch: Die zweitausendjährige jüdische Geschichte Europas steht damit im Rückblick von heute zwangsläufig im Zeichen des Holocaust. Es werden Parallelen von den mittelalterlichen Verfolgungen über den Antisemitismus des 19. Jahrhunderts bis hin zum Nationalsozialismus gezogen. Jüdische Geschichte erscheint so als Vorgeschichte des Holocaust (Liepach et al. 2014: 176f.). Natürlich ist die Vermittlung des Holocaust ein wichtiger Bestandteil des Geschichtsunterrichts und muss es auch bleiben. Doch werden Jüdinnen und Juden stets als Verfolgte gesehen und nur selten als fester Bestandteil der deutschen Gesellschaft, bleiben alte Vorurteile bestehen und können nicht adäquat aufgearbeitet werden.

\section{Von der Idee zur Umsetzung: Das Projekt Geschichtomat}

Die aufgezeigten Schwierigkeiten in der Vermittlung jüdischer Geschichte führten zur Suche nach alternativen Lehr- und Lernmöglichkeiten. Ivana Scharf vom Atelier für Gesellschaftsgestaltung in Hamburg entwickelte schließlich 2013 das Projekt Geschichtomat. Damit sollten Jugendliche die Möglichkeit erhalten einen breiten Einblick in die jüdische Geschichte und Kultur ihrer Nachbarschaft zu bekommen und diese selbst zu erforschen. Die Einbindung digitaler Medien sollte das Projekt für die Schüler/innen zeitgemäßer und attraktiver gestalten. Gleichzeitig sollte durch die Einbettung der erarbeiteten Beiträge auf der Geschichtomat Website ihre Sichtbarkeit sowie ihre Nachhaltigkeit erhöht werden. Die Entstehung eines digitalen Stadtplans zur jüdischen Geschichte Hamburgs aus der Sicht von Jugendlichen für Jugendliche war das Ziel.

Getragen wird Geschichtomat vom Hamburger Institut für die Geschichte der deutschen Juden. Nach knapp zweijähriger Entwicklungsphase konnte das Projekt im Februar 2013 erstmalig als Modell an einer katholischen Gesamtschule in Hamburg-Barmbek realisiert werden. Seitdem haben 40 Projektwochen (Stand Juli 2019) an verschiedenen Schulen im Hamburger Stadtgebiet stattgefunden, darunter vor allem sogenannte Stadtteilschulen (Gesamtschulen mit gymnasialem Zweig), aber auch Gymnasien und regionale Berufs- und Bildungszentren (Förderschulen). Über 700 Schüler/innen von der 7 . bis zur 12. Schulstufe haben sich bislang beim Geschichtomat beteiligt: Sie waren auf jüdischen Friedhöfen, haben koschere Gummibärchen verkostet, Matzen gebacken oder die Synagoge besucht. Biografien aus rund 400 Jahren jüdischer Geschichte in Hamburg wurden erforscht. Die Jugendlichen haben sich mit dem Schicksal verfolgter Jüdinnen und Juden im Nationalsozialismus auseinandergesetzt, 
sich mit den Kindertransporten oder der Bücherverbrennung von 1933 beschäftigt. Dafür haben die Schüler/innen Gedenkstätten, Museen, Archive und Stadtteilinitiativen besucht, dort gefilmt und Interviews mit Historiker/innen und anderen Expertinnen und Experten geführt. In den vergangenen sechs Jahren entstanden über 180 Kurzfilme. Sie alle sind online abrufbar auf der Projektwebsite www.geschichtomat. de. Dort wurde jeder Beitrag mit einer Koordinate auf einem digitalen Hamburger Stadtplan verknüpft.

Die Einbindung von Geomedien ist ein Ziel des Projektes Geschichtomat: Der bereits bestehende und ständig wachsende digitale Stadtplan der Hansestadt Hamburg zu Orten ehemaligen und historischen jüdischen Lebens soll die vielfältige jüdische Geschichte der Stadt sichtbar und erfahrbar machen. Ein Ansatz der sich aktuell bei einigen Projekten im Rahmen der Holocaust Education wiederfinden lässt (Wöhs et al. 2018: 193). Archivmaterialien, Bilddatenbanken und Gedenkorte sind zunehmend auch online einsehbar bzw. aufsuchbar. Geschichtomat bietet die Möglichkeit, sich online einen Überblick über spezielle Themen und Orte zu verschaffen. Genauso gut kann der Ort aber auch „live” aufgesucht und nähere Informationen dazu online abgerufen werden.

\section{Wie funktioniert Geschichtomat? Eine Projektwoche}

Vor Beginn der eigentlichen Projektwoche finden in der Regel ein bis zwei Treffen mit der teilnehmenden Schulklasse und der Lehrkraft statt. Diese sogenannten Auftaktveranstaltungen dienen zum gegenseitigen Kennenlernen, zur Erklärung des Projekts und des Ablaufs der Projektwoche sowie zur Überprüfung des Wissenstands und eventueller Vorkenntnisse und Interessen der Schüler/innen. Bei den Vorkenntnissen wird nicht nur historisches Wissen abgefragt. Es geht auch darum, ob die Jugendlichen bereits filmisch tätig waren. Haben sie schon einmal gedreht, vielleicht sogar einen Film geschnitten?

Die Jugendlichen werden während der Auftaktveranstaltung gebeten sich in Gruppen zusammenzufinden und Mindmaps zum Thema "Jüdische Geschichte" zu erstellen. Diese werden im Anschluss präsentiert und gemeinsam ausgewertet bzw. ergänzt. Diese Vorgehensweise bietet die Möglichkeit Schwerpunkte im Wissen der Schüler/innen festzustellen, aber auch auf bestimmte Fehlinformationen oder Vorurteile eingehen zu können und diese aufzulösen. Die meisten bisher erstellten Mindmaps bestanden zu einem großen Teil aus Begriffen, die sich mit dem Thema Holocaust befassten. Gängige Begriffe waren dabei „Hitler“, „Auschwitz“, „Gas“, „Verfolgung“ und „Ermordung“.
Der Nationalsozialismus wird in Deutschland ab der 9. bzw. 10. Klasse (15./16. Lebensjahr) unterrichtet. Doch auch deutlich jüngere Schüler/innen wählen ähnliche Begrifflichkeiten. Sicherlich spielen hier Medien, wie Film, Fernsehen und YouTube eine große Rolle. Hier schnappen die Jugendlichen erste Informationen über das Thema auf. Neben dem Thema Holocaust lassen sich einige religiöse Begriffe auf den Mindmaps wiederfinden. Insbesondere die jüngeren Schüler/innen erinnern sich noch an das Thema Judentum im Religionsunterricht, welches meist in der 5. oder 6. Klasse (10.-12. Lebensjahr) durchgenommen wird. Aber auch gängige Vorurteile lassen sich auf den Mindmaps lesen: „Rothschild“, „Banken“, „Wirtschaftskrise“. An diesem Punkt ist es wichtig mit den Schüler/innen zu sprechen. Was verbinden sie mit diesen Begriffen? Warum haben sie diese aufgeschrieben? Woher stammen ihre Informationen? Ein Thema kommt dabei fast nie zum Tragen: der Nahostkonflikt. Es scheint, dass die wenigsten Schüler/innen Kenntnisse über diesen Konflikt besitzen oder ihn zumindest nicht automatisch mit deutsch-jüdischer Geschichte verknüpfen.

Am Ende der Auftaktveranstaltung werden den Jugendlichen die verschiedenen Themen ihrer Projektwoche vorgestellt. Maximal fünf Schüler/innen finden sich jeweils in einer Gruppe zusammen und wählen ein Thema aus. So entstehen pro Projektwoche vier bis fünf unterschiedliche Beiträge. Bisher wurde jedes Thema nur ein einziges Mal von einer Schüler/innengruppe bearbeitet. Die meisten Themen besitzen einen Stadtteilbezug und befinden sich im direkten Umfeld der teilnehmenden Schule. Lokalgeschichte spielt bei der Themenwahl eine enorme Rolle. Ausnahmen sind allerdings möglich. So gibt es Lehrkräfte, die sich spezielle Themen wünschen. Teilweise ist die Projektwoche auch in den aktuellen Geschichtsunterricht eingebunden. So fanden Projektwochen zum Deutschen Kaiserreich, zur Wirtschaftsgeschichte oder zum Ersten Weltkrieg statt.

In Hamburg findet während dieser Auftaktveranstaltung noch eine Rallye durch das Grindelviertel, das ehemalige jüdische Viertel der Stadt, statt. Die Schüler/innen bekommen so die Möglichkeit, frühere und heutige Orte jüdischen Lebens zu erforschen und einen ersten Eindruck von der jüdischen Geschichte ihrer Heimatstadt zu gewinnen. Die Ergebnisse der Rallye werden gemeinsam besprochen und präsentiert. Inzwischen gibt es die Grindelrallye auch zum Download auf der Geschichtomat-Website. So kann sie von Lehrkräften auch außerhalb des Projektes durchgeführt werden.

Geleitet wird jede Projektwoche von einem Team aus zwei Medienpädagoginnen und Medienpädagogen sowie einem Kulturvermittler oder einer Kulturvermittlerin. Diese unterstützen die teilnehmenden 
Schüler/innen sowohl im Umgang mit der Technik und dem Schnitt als auch beim inhaltlichen Verstehen und Erarbeiten der Themen. Das benötigte technische Equipment, wie Kameras, Ton und Schnittrechner (MacBooks inklusive der Schnittsoftware Final Cut) wird zudem vom Geschichtomat-Team mit in die Schule gebracht. Dies ist eine über die Jahre bewährte Vorgehensweise. Viele Schulen verfügen nur über eine geringe technische Ausstattung. Zudem sind die Geräte vielfach schlecht gewartet, so fehlen z. B. nötige Updates. Insbesondere im Fall von schulweiten Projektwochen sind Schulkameras und ähnliches Equipment schnell vergriffen.

Die einzelnen Themen werden im Vorfeld von der Projektleiterin recherchiert. Sie stellt Materialien zusammen und schaut, ob sich ein Thema auch visuell umsetzen lässt, ob es z. B. noch Bildmaterial gibt oder einen aktuellen Ort, der gefilmt werden kann. Zudem sucht sie gegebenenfalls nach Expertinnen und Experten sowie Ansprechpartner/innen für Interviews, wie z. B. Historiker/innen, Gemeindemitgliedern oder Mitarbeiter/innen von Stadtteilinitiativen und Geschichtswerkstätten. Diese besitzen gegebenenfalls noch Dokumente und Bildmaterialien, beantworten den Jugendlichen ihre Fragen und können helfen ein Thema verständlich zu erklären. Wünschenswert wäre es, wenn Teile dieser Vorarbeiten von den Jugendlichen selbst geleistet werden könnten. Idealerweise könnten sie sich selbstständig mit ihrer Nachbarschaft auseinandersetzen und überlegen, welche Themen sie spannend und für erarbeitungswürdig halten. Auch das eigene arrangieren von Interviewpartner/innen und Drehterminen könnte eine wichtige Lernübung für Schüler/innen sein. Der enge Zeitplan des Geschichtomat-Projektes lässt diese selbstständigen Vorarbeiten jedoch nicht zu. Für die meisten Schulen bzw. Lehrkräfte ist es bereits eine Herausforderung eine Schulklasse für eine gesamte Woche aus dem Unterricht zu nehmen. Viele Schule besitzen zwar Zeit für Projektarbeit, über eine Woche geht diese aber nicht hinaus.

$\mathrm{Zu}$ Beginn der Projektwoche erhalten die teilnehmenden Jugendlichen verschiedene Materialien, darunter Texte und Bildmaterial zur Einarbeitung in ihr Thema. Die thematische Bandbreite ist dabei groß: Ein nahegelegener Straßenname oder ein Stolperstein können zu einer Biografie führen, ein Denkmal erzählt eine Geschichte, ein jüdischer Feiertag fällt in die Zeit der Projektwoche. Teilweise befinden sich noch frühere oder auch aktuelle Orte jüdischen Lebens in der Nähe der Schule, wie z. B. eine jüdische Schule, eine Synagoge oder auch ein Friedhof. Zudem gibt es allgemeinere Themen, wie etwa die jüdische Geschichte eines bestimmten Stadtteils.

Der erste Tag einer Projektwoche beginnt aber auch mit Organisatorischem und der Planung der restli- chen Woche. Die Jugendlichen erhalten eine Übersicht, ob und wann sie Interviews haben und wer ihre Interviewpartner/innen sind. Diese Interviews müssen vorbereitet, die Fragen erarbeitet und es müssen gegebenenfalls passende Drehorte gesucht werden. Bereits am ersten Projekttag ist es wichtig, dass sich die Schüler/innen einen Überblick über ihre Arbeit verschaffen. Ein sogenanntes Drehbuch soll dafür erstellt werden. Dieses beinhaltet Ideen für den späteren Beitrag, z. B. welches Bildmaterial verwendet werden soll, welche Orte besucht und gefilmt werden und ob Schüler/innen selbst vor eine Kamera treten.

Parallel findet für jede Schülergruppe eine Einführung in die Technik durch die Medienpädagoginnen und -pädagogen statt. Für viele Schüler/innen ist es der erste Umgang mit einer professionellen Kamera. Das Einstellen und Aufbauen der Technik will geübt sein, aber auch das Platzieren eines Interviewpartners oder einer -partnerin muss erlernt werden.

Der zweite und dritte Tag der Projektwoche dienen dem Sammeln von Materialien. Interviews werden geführt, Fotos gemacht, Ansager vor der Kamera eingesprochen. Während ihrer Recherche lernen die Schüler/innen, unter anderem durch unterschiedliche Primärzeugnisse oder Interviews mit Zeitzeuginnen und Zeitzeugen sowie Expertinnen und Experten, verschiedene Sichtweisen auf die Vergangenheit kennen (Bergmann 2008: 160).

Durch die Arbeit an einem eigenen Projekt ist Lernen nicht nur eine passive Aufnahme von Wissen, sondern ein aktiver, gestaltender Prozess, in dem die/ der Lernende das zu Lernende auf der Basis von Vorwissen und Vorerfahrungen individuell „konstruiert“. Forschendes Lernen kann nur in Projekten stattfinden. Die Schüler/innen arbeiten mit Quellen, führen Zeitzeugen- und Zeitzeuginneninterviews und gelangen so zu eigenen, vielleicht auch neuen, Befunden. Diese Form der Auseinandersetzung mit Geschichte verlangt nach einer längerfristigen und kontinuierlichen Beschäftigung mit einem Thema. In der Regel überschreitet dies die übliche Organisationsform der Schule (Sauer 2014: $11 \mathrm{ff}$.). Deswegen ist es sinnvoll, sich externe Bildungsprojekte für eine Projektwoche oder einen abgesteckten Zeitraum an die Schule zu holen. Diese bringen das nötige Fachwissen und, wie im Fall des Projektes Geschichtomat, auch die benötigte Ausstattung mit.

Außerschulische Lernorte spielen bei Geschichtomat eine große Rolle. Die Jugendlichen erforschen ihren Stadtteil und treten dabei in Kontakt mit den verschiedensten Einrichtungen. Museen, Archive, Gedenkstätten, aber auch Stadtteilinitiativen, kirchliche Vereinigungen sowie Nachbarinnen und Nachbarn, und Zeitzeuginnen und Zeitzeugen sind wichtige Ansprechpartner/innen für die Jugendlichen. Hierdurch 
können Kontakte entstehen, die auch nach Abschluss der Projektwoche von Schulen, Lehrenden, aber auch den Jugendlichen selbst genutzt werden. Bildungslandschaften können entstehen und die Stadt selbst wird zu einem Bildungsort (Schroer \& Wilde 2010: 182).

Durch die Verankerung der zu bearbeitenden Themen in der eigenen Nachbarschaft, erhalten die Schüler/innen gleichzeitig einen persönlichen Bezug. So ist das Projekt Stolpersteine, welches in Hamburg stark verankert ist, ein Beispiel für diesen persönlichen Bezug. Hamburg besitzt derzeit über 6000 der Gedenkplatten, die in die Gehwege eingelassen sind und an Opfer des Nationalsozialismus erinnern. Zudem erscheinen seit zehn Jahren ausführliche Biografien zu den einzelnen Personen, die von der Hamburger Landeszentrale für politische Bildung herausgegeben werden (Meyer 2006: 9 f.). Die meisten Jugendlichen passieren auf ihrem Schulweg mehrere der Gedenksteine. Die Biografien hinter den Namen sind ihnen jedoch unbekannt. Eine einzelne Biografie selbstständig zu erforschen und zu verstehen, dass in ihrer unmittelbaren Umgebung jemand lebte, der im Nationalsozialismus verfolgt und ermordet wurde, bietet ihnen einen anderen, einen individuelleren und teilweise auch leichter verständlicheren Zugang zur Geschichte des Holocaust.

Insbesondere an den Drehtagen ist es wichtig, dass das Geschichtomat-Team aus drei Personen besteht. Jüngeren Schüler/innen ist es z. B. vielfach nicht gestattet das Schulgelände allein zu verlassen, was zum Sammeln von Bildmaterial in der Regel unerlässlich ist. Bei einem Interview wird generell die Gruppe von einem Geschichtomat Teammitglied begleitet, um den Aufbau der Technik und das einwandfreie Funktionieren dieser zu sichern.

Der Schnitt der Videos dauert in der Regel am Längsten. Hierfür müssen mindestens anderthalb Schultage eingerechnet werden. Die Interviews müssen wieder und wieder angehört, wichtige Informationen herausgefiltert und Zusammenhänge verstanden werden. Damit findet noch einmal eine tiefere inhaltliche Auseinandersetzung mit dem gewählten Thema statt. Die Schüler/innen sollen lernen eine Geschichte zu erzählen. Dabei setzen sie sich mit ihrer Nachbarschaft auseinander. Geschichte wird bei Geschichtomat erarbeitet, denn die Jugendlichen sollen einen eigenen Beitrag erstellen und eine eigene Geschichte erzählen. Dafür müssen sie die verschiedenen Informationen, die sie über die letzten Tage gesammelt haben, filtern, ordnen und in ihrem Beitrag strukturieren. Durch die Auswahl dessen, was in das spätere Video soll, lernen die Schüler/innen das Geschichte immer eine Narration zugrunde liegt. Es muss also immer hinterfragt werden, nach welchen Kriterien und auf welcher Grundlage Informationen ausgewählt wurden (Barricelli 2012: 261).
Nicht jede/r Schüler/in besitzt ein ausgeprägtes Interesse an Geschichte. Teilweise liegt dies vielleicht an einem generellen Desinteresse am Thema oder aber an veralteten Vermittlungsformen. Mit der Aussicht auf die Produktion eines Videos und den Einsatz digitaler Medien, werden vielfach auch diese Schüler/innen für das Projekt gewonnen. Um aber den späteren Beitrag erarbeiten zu können, muss zwangsläufig eine intensive Auseinandersetzung mit dem eigenen, kleinen historischen Thema erfolgen. So lassen sich oftmals Interessen für Geschichte wecken, die vorher nicht existierten. Die Aussicht auf eine Arbeit mit digitalen Medien bietet für einige den Anreiz sich letztlich auch mit Geschichte zu beschäftigen.

In der Regel haben die Schüler/innen selten mit einem professionellen Schnittprogramm gearbeitet. Vereinzelt ist auf Smartphones gefilmt und geschnitten worden. Einige Jugendliche haben bereits bei YouTube Videos hochgeladen. Für Geschichtomat sollen aber kurze Dokumentationen entstehen, die sachlich korrekt sein müssen. Die späteren Beiträge müssen jedoch nicht nur inhaltlich richtig sein, sie sollen auch visuell ansprechen. Um die Aufmerksamkeit späterer Nutzer/innen zu sichern, sollen die Videoclips eine Länge von vier bis fünf Minuten haben. Spannung muss in ihnen aufgebaut werden, sie sollten abwechslungsreich gestaltet werden und gegebenenfalls unterschiedliche Medien, wie zum Beispiel Musik oder Fotos, einbinden (Kowitz-Harms \& Menny 2014: 335). Gleichzeitig dürfen die Jugendlichen keiner „Technikfaszination" erliegen. Der Inhalt darf bei aller gestalterischen Freiheit nicht zu kurz kommen. Das dies nicht geschieht, liegt in der Verantwortung der Geschichtomat-Mitarbeiter/innen und der Lehrkraft.

Zum Abschluss einer Projektwoche werden die fertigen Beiträge auf die Geschichtomat-Website hochgeladen. So erhalten die Jugendlichen die Möglichkeit, ihre Ergebnisse direkt online anzusehen und diese ihren Freunden und ihrer Familie zu präsentieren (vgl. Abb. 1). Die Veröffentlichung der Beiträge auf einer Website verleiht ihnen eine höhere Wertigkeit gegenüber einer Präsentation im Klassenraum. Gleichzeitig erlaubt die niedrigschwellige Publikationsmöglichkeit den Jugendlichen selbst die Rolle von Geschichtsproduzentinnen und -produzenten einzunehmen und bietet Raum für eigene Gestaltung und Selbstdarstellung. Aber die Veröffentlichung von Beiträgen im Internet birgt auch Herausforderungen. Die Verbreitung der Videos ist kaum kontrollierbar. Geschichtomat-Beiträge sind deshalb auf einem nicht-öffentlichen Kanal bei YouTube eingebunden. So können sie auf dieser Plattform nicht direkt gefunden und ausschließlich über die Geschichtomat-Website aufgerufen werden. Eine Weiterverbreitung und Verlinkung werden somit erschwert. 


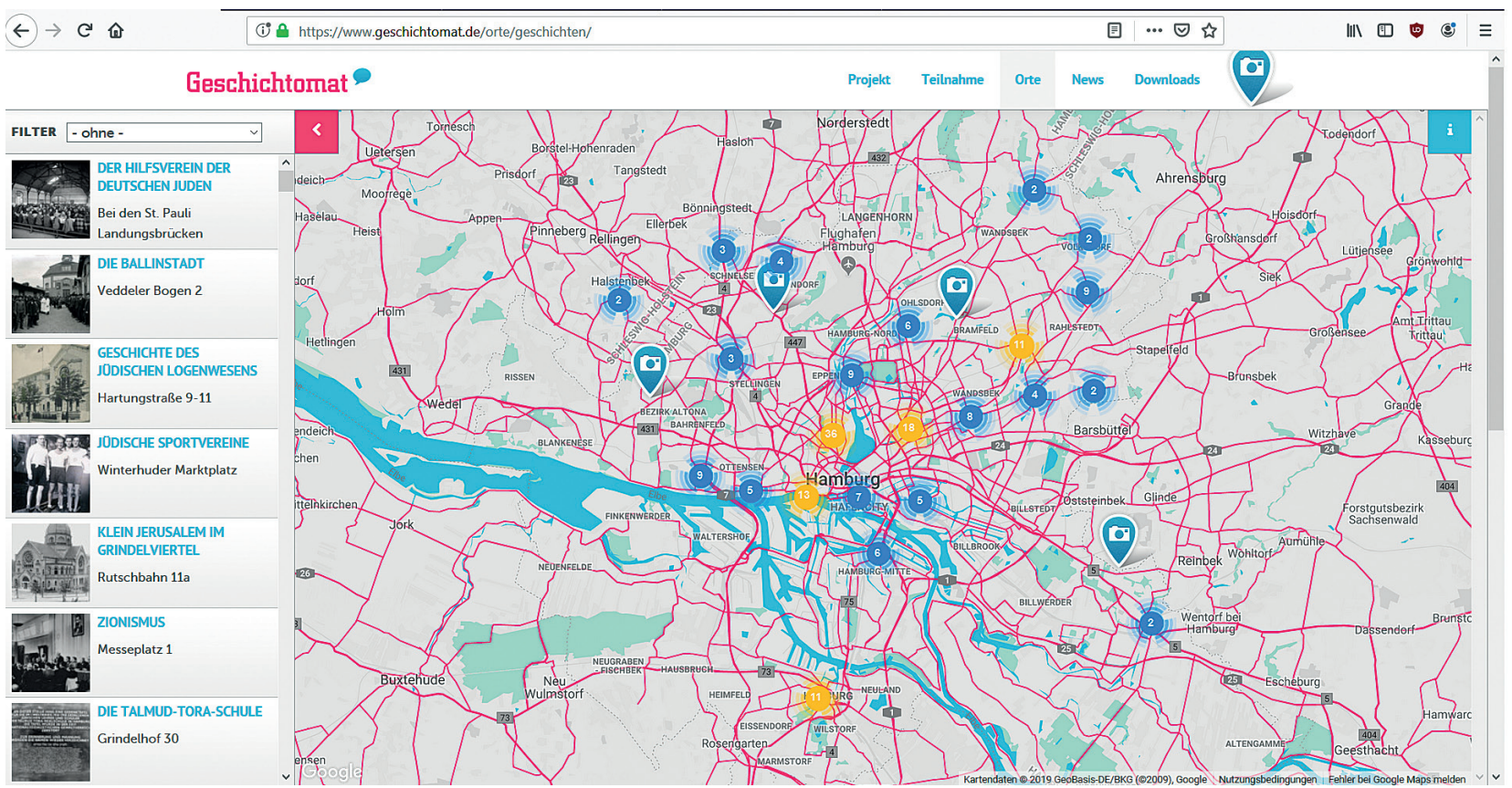

Abb. 1: Der digitale Stadtplan auf www.geschichtomat.de; (C) Geschichtomat, Juni 2019

Neben der Veröffentlichung auf der Website findet zum Abschluss jeder Projektwoche noch eine klassische Präsentation der Ergebnisse im Klassenraum statt. Die Gruppen stellen ihre Filme entweder einander oder auch weiteren Klassen vor, erklären ihr Thema und berichten von Schwierigkeiten wie auch Erfolgen während der Projektwoche. Dies bietet die Möglichkeit den Jugendlichen direkt Feedback zu ihrer Arbeit zu geben.

\section{Fazit: Digitale Bildung trifft auf Lokalgeschichte}

Wissen kann nicht beigebracht oder vermittelt werden, sondern muss individuell erworben werden. Dabei ist es wichtig, dass Lernen, auch historisches, einen Bezug zur Alltagssituation der/des Lernenden haben muss. Diesen Bezug bietet die Lokalgeschichte. In Kombination mit digitalen Medien greift sie die Lebenswirklichkeit der Jugendlichen auf und kann zu einem gesteigerten Interesse sowie einem leichteren Zugang zu Geschichte führen.

Die Einbindung digitaler Medien in den Unterricht bedeutet die Einbindung der Lebenswelt der Jugendlichen. Geschichte begegnet ihnen nicht mehr nur in Büchern, sondern im Netz, so zum Beispiel auf YouTube, Facebook, Instagram und in Computerspielen. Die Aufgabe der Schule ist es, die Schüler/ innen kompetent zu machen, für die Auseinandersetzung mit Geschichte jenseits des Unterrichts. Daher führt die Einbindung digitaler Medien zu einer Kompetenzorientierung der Schüler/innen. Die eigenen digitalen Angebote, wie zum Beispiel Videos, werden zum Erstellen von „Digital Storytelling" verwendet. Es entstehen neue Formen historischer Narration, durch den Einsatz von Text, Ton und Bildmaterial. Gleichzeitig beginnt die Auseinandersetzung mit der Frage, wie welches Element genutzt wird (Bernsen et al. 2012: 12).

Das Gelernte wird verankert, wenn es nacherzählt und präsentiert wird, sich der Nachfrage, der Kritik und der Bestätigung stellt. Außerdem wird Lernen durch gemeinsame Aktivitäten mit anderen und durch wechselseitiges Lehren und Lernen unterstützt. Durch Handlungen, eigene Erfahrungen und Kreativität wird es erfolgreicher und auch Emotionen helfen beim Lernen (Wenzel 2017: 9 ff.).

Die an Geschichtomat teilnehmenden Jugendlichen erforschen und entdecken ihre Nachbarschaft und lernen jüdische Geschichte auf eine neue, einzigartige Weise kennen. Dadurch entwickeln sie einen Bezug zur jüdischen Kultur, Geschichte und zu jüdischem Leben, der ihnen durch den normalen Schulunterricht nicht vermittelt werden kann. Sie erhalten die Chance sich in die regionale Geschichtspolitik einzumischen. Die ist auf lokaler Ebene gar nicht so schwer. So stecken konträre Vergangenheitsdeutungen in Straßennamen, Denkmalstiftungen und Denkmalpflegeprojekten. Ansprechpartner/innen und eine interessierte Öffentlichkeit finden sich zahlreich auf lokaler Ebene. Kommunalpolitiker/innen, Museumsleiter/innen, Archivdirektor/innen und Mitarbeiter/ innen der Stadtverwaltung oder Vereinsvorstände sind als kulturpolitische Entscheidungsträger/innen für Jugendliche ansprechbar (vgl. John 2019). 
Hier knüpft das Projekt Geschichtomat an, indem es den alltäglichen Umgang der Jugendlichen mit dem Internet nutzt und ihnen gleichzeitig einen adäquaten Umgang mit dem Medium Internet vermittelt. Zielgerichtete Recherchen werden eingeübt und der Blick für die Gefahren und Probleme des Internets eingeschärft. So müssen sich die Teilnehmer/innen mit schwierigen Aspekten wie Persönlichkeits- und Urheberrechten und dem nicht vorhandenen „Recht auf Vergessen" auseinandersetzen. Die Vermittlung jüdischer Geschichte und Kultur kann zugleich zu einer Sensibilität gegenüber problematischen Inhalten sowie den Grenzen des Sag- und Darstellbaren führen.

Im Bereich der Geomedien gibt es den Ansatz einer Education for Spatial Citizenship, welche Schüler/innen im Unterrichtsgeschehen zu einem mündigen Umgang mit digitalen Geomedien im Kontext gesellschaftlicher Aushandlungsprozesse befähigen will (Jekel et al. 2015: 7). Geschichtomat verfolgt eine ähnliche Vorgehensweise lediglich für das Fach Geschichte: Schüler/innen sollen zum einen den Umgang mit digitalen Werkzeugen lernen sowie das Recherchieren und den kritischen Umgang mit gefundenen Online-Quellen einüben. Eine Vorgehensweise, die jede/r Geschichtsstudent/in im Studium im Umgang mit gedruckten Quellen beigebracht bekommt.

Die Chancen und Möglichkeiten Lokalgeschichte in den Unterricht zu integrieren sind groß. Es kommt aber darauf an, die Interessantheit zu vermitteln und die Eigenheit einer quellennahen und besonders partizipativen Art historischen Denkens auszuschöpfen. Die Einbeziehung digitaler Medien kann helfen, das Interesse von Schüler/innen neu zu wecken, auch für die Lokalgeschichte, und die Attraktivität von historischem Lernen und historischen Orten zu erhöhen.

Für die Zukunft wird es spannend sein zu sehen, inwieweit Online-Projekte wie Geschichtomat auch weiterführend genutzt werden. Die Anfragen für Teilnahmen an Projektwochen sind in Hamburg hoch. Bis zu acht können pro Jahr umgesetzt werden. Die Rückmeldungen der Lehrkräfte wie auch der teilnehmenden Jugendlichen sind grundsätzlich positiv. Die erarbeiteten Videos könnten für den Unterricht, für Stadtgänge und andere Bildungsprojekte genutzt werden. Doch die Nutzerzahlen der Website sprechen eine andere Sprache: An den meisten Tagen wird die Website von kaum mehr als zehn Besuchern aufgerufen (vgl. Google Analytics). Auch die bereits vorhandenen Unterrichtsmaterialien werden nur selten heruntergeladen. Die Erhöhung der Sichtbarkeit der Website sowie die Nutzung der bereits erarbeiteten Beiträge sind wichtige aktuelle, wie auch zukünftige Aufgaben.

\section{Literatur}

Barricelli, M. (2012): Narrativität. In: Barricelli, M. \& M. Lücke (Hrsg.): Handbuch Praxis des Geschichtsunterrichts. Wochenschau Verlag, Schwalbach/Ts. S. 255-280.

Bergmann, K. (2008): Geschichtsdidaktik. Beiträge zu einer Theorie historischen Lernens. Wochenschau Verlag, Schwalbach/Ts.

Bernsen, D., König, A. \& T. Spahn (2012): Medien und historisches Lernen: Eine Verhältnisbestimmung und ein Plädoyer für eine digitale Geschichtsdidaktik. In: Zeitschrift für digitale Geschichtswissenschaft 1. S. 1-27.

Freie und Hansestadt Hamburg, Behörde für Schule und Berufsbildung (2014): Bildungsplan Stadtteilschule, Jahrgangsstufen 7-11, Geschichte. https://www.hamburg.de/contentblob/4327780/0e42f20dca0ff325631f6 0a42ae79543/data/geschichte-sts.pdf (29.07.2019)

Hunner, J. (2011): Historic Environment Education. Using Nearby History in Classrooms and Museums. In: The Public Historian 33(1). S. 33-43.

Jekel, T., Gryl, I. \& A. Oberrauch (2015): Education for Spatial Citizenship: Versuch einer Einordnung. In: GW-Unterricht 137. S. 5-13. http://www.gw-unterricht.at/images/pdf/ gwu_137_05_13_jekel_gryl_oberrauch.pdf (29.07.2019)

John, A. (2019): Lokales Geschichtswissen statt „Lebendige Lokalgeschichte“. http://lernen-aus-der-geschichte.de/ Lernen-und-Lehren/content/14354 (30.04.2019)

John, A. (2018): Lokal- und Regionalgeschichte. Wochenschau Verlag, Frankfurt am Main.

Kowitz-Harms, St. \& A. Menny (2014): Schülerprojekt Geschichtomat. Zur Vermittlung jüdischer Geschichte im Internet. In: Demokratische Geschichte 25. S. 329-342.

Landesinstitut für Lehrerbildung und Schulentwicklung Hamburg (2011): Bildungsplan Gymnasium, Sekundarstufe I, Geschichte. https:/www.hamburg.de/contentblob/2373302/b08baff8e839e62a31d6dbf07868c762/ data/geschichte-gym-seki.pdf (29.07.2019)

Liepach, M. \& W. Geiger (2014): Fragen an die jüdische Geschichte. Darstellungen und didaktische Herausforderungen. 2. Aufl., Wochenschau Verlag, Schwalbach/Ts.

Meyer, B. (2006): Die Verfolgung und Ermordung der Hamburger Juden 1933-1945. Geschichte. Zeugnis. Erinnerung. Landeszentrale für politische Bildung, Hamburg.

Sauer, M. (2014): Projekt und Projektarbeit in Geschichte. In: Sauer,M.(Hg.):Spurensucher.EinPraxisbuchfürhistorische Projektarbeit. edition Körber Stiftung, Hamburg. S. 9-30.

Schroer, M. \& J. Wilde (2010): Ort. In: Reutlinger, Ch., Fritsche, C. \& E. Lingg (Hrsg.): Raumwissenschaftliche Basics. Eine Einführung für die Soziale Arbeit. VS Verlag, Wiesbaden. S. 181-190.

Schwabe, A. (2018): Womit? (Digitale) Medien des historischen Lernens. Einführung in die Sektion. In: Sandkühler, T., Bühl-Gramer, Ch., John, A., Schwabe, A. \& M. Bernhardt (Hrsg.): Geschichtsunterricht im 21. Jahrhundert. Eine geschichtsdidaktische Standortbestimmung. V \& R unipress, Göttingen. S. 413-423.

Wenzel, B. (2017): Kreative und innovative Methoden. Geschichtsunterricht einmal anders. 7. Aufl., Wochenschau Verlag, Frankfurt am Main.

Wöhs, K., Paulischin-Hovdar, R., \& A. Gatterbauer (2018): Fostering Holocaust Education and Remembrance Culture using Geomedia. In: GI Forum 6(2). S. 193-206. 